\title{
CONSTRUCCIÓN SOCIAL DE LA COMUNIDAD PARA EL DESARROLLO DEL CALLEJÓN DE HUAYLAS, CARAZ
}

\section{SOCIAL CONSTRUCTION OF THE COMMUNITY FOR THE DEVELOPMENT OF THE ALLEY OF HUAYLAS, CARAZ}

\author{
MILDRED PAREDES TARAZONA ${ }^{1}$, JESÚS MARÍA DE MIGUEL CALVO ${ }^{2}$, MARIA JOSÉ \\ CARBAJAL ITURRY, MIRIAM LAGUNA SANCHEZ. \\ Facultad de Psicología UNMSM \\ ${ }^{2}$ Universidad Autónoma de Madrid en el Centro Universitario de Salud Pública.
}

\section{RESUMEN}

OBJETIVO: Describir las dimensiones psicosociales de la construcción organizacional de la comunidad andina, tanto en las Comunidad Campesina Cruz de Mayo (caseríos: Pampacocha, Queral, Miramar y Chinchay) como en la Comunidad Cajabamba Alta. MATERIAL y MÉTODOS: Muestra de 97 sujetos mayores de 16 años por caserío. Se empleó el cuestionario CREO, adaptado, se utilizó el análisis discriminante, y el Chi cuadrado de Pearson. RESULTADOS: En la escala ordinal de 4 puntos, en la imagen, la Productividad es la variable más valorada (3.15) y la menos valorada la Retribución (2.14); y para la satisfacción, la identidad (3.17) en oposición a la Accidentabilidad (1.85); Los coeficientes estandarizados de las funciones discriminantes canónicas indican la peculiaridad de cada comunidad, este mismo posibilita clasificación correctamente a los sujetos de una comunidad u otra con una probabilidad de $97.8 \%$ y por caserío al $100 \%$.

CONCLUSIONES: Cada comunidad construye y comparte una representación de la comunidad que es sui generis; el CREO es un instrumento válido para analizar la representación colectiva que comparten los comuneros sobre su comunidad A diferencia del Sexo, la Pertenencia a un caserío es la variable de agrupación con mayor poder para diferenciar la imagen que tienen los miembros de la comunidad, porque el caserío posibilita y determina el intercambio intersubjetivo que cimienta la representación compartida y socialmente construida de la comunidad. El bajo número de variables que las diferencie sugiere la existencia de un alto índice de identidad con la comunidad.

Palabras Clave: Construcción social, Diversidad, Identidad, Dimensiones psicosociales de la comunidad andina, Cuestionario CREO.

\footnotetext{
ABSTRACT

OBJECTIVE: Describe the psycho social dimensions of the organizational construction of the Andean communities Cruz De Mayo, Pamapacocha, Queral, Miramar and Cajabamba. Determine the elements of identity and diversity that create the perception that the people have about their community. MATERIAL AND METHOD: Ninety Seven individuals were interviewed sixteen years of age or older with the translated Creo $\mathrm{C}$ questionnaire. A discriminate analysis was used to evaluate the translated forms of the Creo $\mathrm{C}$ questionnaire. The Chi Cuadrado De Pearson was used to evaluate the results from the questionnaire after interviewing was complete. RESULTS: Using a scale from one to four, in perception, productivity was the more important element that scaled 3.15 opposed to retribution at 2.14. When interviewed about their satisfaction, identity was higher at 3.17 opposed to

${ }^{1}$ Email: mparedest@unmsm.edu.pe
} 
hazard at 1.85. The standardized coefficients from the discriminative function indicate that each community is particular and helps distinguish individuals from one community to another by $97 \%$ to $100 \%$, enough for certainty. CONCLUSIONS: Each community shares and develops the representation of the community that is particular. The Creo $\mathrm{C}$ is valid to use and analyze the group representation that the individuals have from their communities. The sense of belonging to a small village is the most powerful variable for group representation and to distinguish the perception that the members have about their community. Identity to small villages determine the subjective exchange. The low number of variables that distinguish the community suggests that identity exist in the community.

Keywords: Social construction, Diversity, Identity, Andean communities's psycho social dimensions, Creo $\mathrm{C}$ questionnaire.

\section{INTRODUCCIÓN}

La psicología social se registra dentro de las categorías del saber como una ciencia que persigue, en última instancia, el bienestar y la mejora de los condiciones de vida de las personas y de los pueblos, insistiendo en la potestad de individuos y comunidades para ejercer sus derechos humanos, civiles, políticos, sociales y culturales. Y lo hace desde el conocimiento del comportamiento de las personas y de la comunidad. Su razón de ser se circunscribe, de esta forma, en una irrefutable posición de apuesta e implicación con el desarrollo.

La aportación de la ciencia social en los procesos de desarrollo no debe consistir en una evaluación descriptiva-informativa de las formas básicas de organización social. El innegable valor antropológico de este tipo de descripciones debe conjugarse con la apuesta por una intervención que mejore el bienestar y las condiciones de vida de las personas y las comunidades. Para implantar un plan de mejora, resulta necesaria la capacidad de explicar y de predecir. El conocimiento de la dinámica que estructura el comportamiento social, en y de la comunidad, se hace indispensable para el progreso, tanto como la participación de la población vinculada a talo cual comunidad. Tal y como apunta De Miguel (2004) "si hay vinculación simbólica y emocional hay sociedad" (comunidad en nuestro caso).

El dominio conceptual de las dimensiones psicosociales que explican la organización de las comunidad andina será el objetivo de este proyecto. El estudio se ha planteado desde el análisis de las dimensiones psicosociales que caracterizan a los pobladores del territorio rural de influencia de Caraz, capital de la provincia de Huaylas, perteneciente al departamento de Ancash. El análisis persigue el conocimiento de los elementos de identidad y diversidad que conforman la imagen que tienen los comuneros de su comunidad y el reconocimiento de las relaciones, en términos de actitud, que guardan con su comunidad. También se quieren despejar los elementos de intervención para la mejora de la calidad de vida y el ejercicio efectivo de los derechos de las personas y de los pueblos atendiendo a 1.- la categorización de los elementos de la realidad que estructura su representación y 2.- sus expectativas.

La ejecución del análisis se ha desarrollado mediante la aplicación del instrumento CREO (cuestionario de representación organizacional) previamente traducido y adaptado al Quechua del callejón de Huaylas.

La base del tema se formula desde el modelo de organización, comunidad en nuestro 
caso. Como una construcción social planteado por De Miguel (1999)²: a) Desde una perspectiva institucional las comunidades tienen poder para constituirse como realidades imponiéndose a sus miembros mediante el conocimiento (se concibe como una realidad socialmente adquirida). Y, b) desde una perspectiva a lo institucional los individuos de la comunidad ejercitan un juego simbólico que genera un conocimiento sobre la propia comunidad (se trata, en este caso de una realidad socialmente elaborada/ producida). La constante en ambas perspectivas se ciñe sobre el hecho de que, en todo caso, hablamos de conocimientos socialmente compartidos

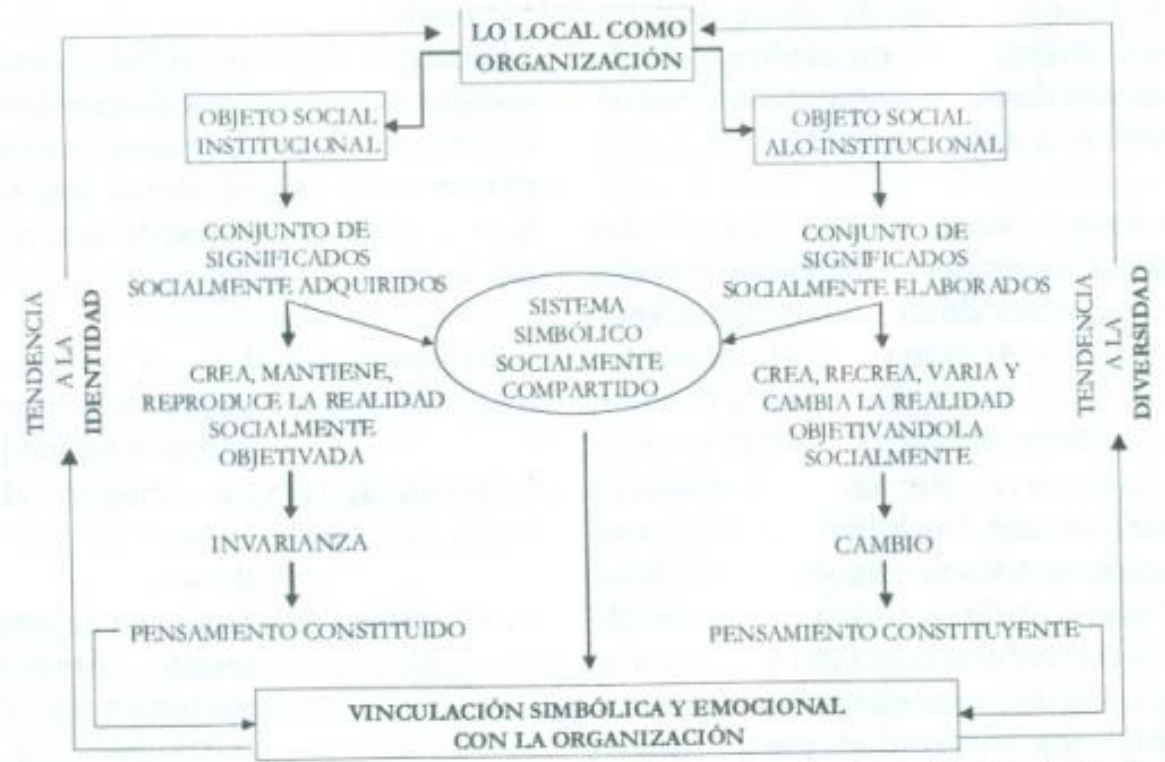

Figura 1.Lo local como organización (De Miguel, 2004, adaptado de De Miguel, 1999)

El modelo se amplía con un método de diagnostico de la comunidad como objeto social, este método de análisis esta caracterizado por:

1. estudiar los componentes de la representación organizacional de la comunidad: a) el conocimiento adquirido, elaborado y compartido sobre la comunidad andina como objeto social b) La estructura de ese conocimiento, c) La actitud que determina la relación de los sujetos de la comunidad andina como objeto.

2. El análisis debe entender a la comunidad andina como realidad objetiva (universo reificado), realidad intersubjetiva (universo consensuado) y realidad subjetiva (universo individual)

3. Participa de un sistema dialectico como método para llegar al conocimiento: a) Los escenarios reales y descritos en la actualidad (tesis), b) los escenarios ideales, futuros y deseados que queremos normalizar (antítesis), c) Los escenarios posibles determinados por las limitaciones propias y las amenazas (síntesis)

4. Utilizar un instrumento validado para el análisis de la representación organizacional (CREO) que se puede adaptar al contexto de las comunidades andinas.

El sistema plantea como ideal un sistema de organización denomina $\mathrm{K}$ resumen, que, se caracteriza por la participación de los miembros de la organización en la generación de 
conocimiento sobre la propia organización y la generación y mejora del conocimiento de experto aplicado a la solución de problemas y toma de decisiones en el trabajo.

Estas características como apuntan M. Paredes y C. González del Río (2003) ${ }^{3}$ son observadas en la comunidad andina por sus valores y creencias. La gestión de la comunidad sustentada en dichos valores

expresa conductas de cooperación, participación, etc, en definitiva: de generación de valor como capital social. (Paredes, M y Arotoma, 1994)4.

Con fundamento en las interpretaciones de la Profesora Paredes sobre la cultura de la comunidad andina (Paredes, M y Arotoma, 1994.; González del Río, C. y Paredes, M., $1999^{5}$ y Paredes, M. y González del Río, C. $2000^{3}$ ) se tiene la expectativa de' que el modelo desarrollado por De Miguel (1999) ${ }^{2}$ y su aplicación al enfoque de desarrollo local (De Miguel, 2004) ${ }^{1}$ aporta una serie de ventajas que lo hacen susceptible de mostrar los aspectos psicosociales más profundos y su dinámica interna. A partir de los resultados de la investigación, se puedan planificar acciones para la mejora del bienestar y la calidad de vida de las personas de las comunidades andinas así como para la ejecución efectiva de los derechos de las personas y los pueblos.

Por ello se plantearon los siguientes objetivos e hipótesis:

a. Realizar un diagnóstico estratégico de variables psicosociales en la Comunidad objeto de estudio.

b. Discriminar los elementos de identidad y los elementos que distinguen a los grupos categorizados en el estudio (diversidad).

c. Plantear un proyecto de desarrollo vinculado a la mejora de las condiciones de vida y la sostenibilidad medioambiental .

d. Contrastar las hipótesis que se presentan a continuación.

\section{Hipótesis:}

La imagen (representación compartida y socialmente construida) que tienen los comuneros de su comunidad está determinada por el objeto representado (comunidad) es el supuesto que plantea la hipótesis 1 :

Hipótesis 1: La imagen de la comunidad es propia de cada comunidad

Hipótesis 1a: La imagen de dos comunidades distintas es distinta.

Hipótesis 1b: Mediante la imagen se puede predecir la pertenencia de un individuo a una determinada comunidad

La imagen se genera por y en la interacción que se produce entre los sujetos de la organización y la interacción está posibilitada por la posición que tienen las personas en la comunidad. Posición definida por cualquier condición social, algunas de ellas son las que hemos incluido en el estudio como variables contextuales, y las categorías incluidas en tales variables delimitan las posiciones analizadas. Este es el supuesto que esboza la redacción de la hipótesis 2:

Hipótesis 2: La imagen se modula por la posición que ocupan los comuneros en la comunidad. Por ello encontraremos diferencias en la imagen en función de la posición ostentada. 
Hipótesis 2a: La imagen está modulada por la pertenencia a un caserío

Hipótesis 2b: La imagen está modulada por la condición

Hipótesis 2c: La imagen está modulada por el sexo

Hipótesis 2d: La imagen está modulada por la edad

Hipótesis 2e: La imagen está modulada por el estado civil

Hipótesis 2f: La imagen está modulada por el nivel de estudios

Hipótesis 2g: La imagen está modulada por el manejo del idioma quechua.

Hipótesis 2h: La imagen está modulada por el manejo del idioma español.

Hipótesis 2i: La imagen está modulada por tener un cargo en la comunidad

\section{MATERIALES Y MÉTODOS}

El territorio donde se ha realizado la investigación se identifica y demarca por cuatro caseríos cuyo común denominador es compartir el puesto de salud. En todos los casos se firmó un acuerdo de colaboración entre los representantes del caserío y los investigadores. Los caseríos dependen políticamente de la comunidad Cruz de Mayo y se denominan Pampacocha, Queral, Chinchay y Miramar. La comunidad Cruz de Mayo, a su vez, se ubica, administrativamente, en el distrito de Caraz, provincia de Huaylas.

Caraz es la población de mayor infiuencia de la zona y capital de la provincia. Desde Caraz, la zona ocupada por los caseríos, se localiza a unos $8 \mathrm{~km}$ lineales con orientación $36^{\circ}$ Norte Las coordinadas de localización de la zona se sitúan aproximadamente en los $77^{\circ}$ $47^{\prime}$ longitud Oeste y $9^{\circ} 00^{\prime}$ de latitud Sur. La población, a fecha julio de 2003, es de 1075 personas distribuidas entre los cuatro caseríos, La población incluida en el estudio está compuesta por los mayores de 16 años, distribuidos como se muestra en la tabla 1:

Tabla 1. Distribución de la población. Fuente: Posta de Salud de Pampacocha.

\begin{tabular}{|c|c|c|c|ccc|ccc|}
\hline Caserío & Sujetos & Familias & $\begin{array}{c}\text { Mayores de 16 } \\
\text { años }\end{array}$ & \multicolumn{2}{|c|}{ Muestra e=O.05 } & \multicolumn{3}{|c|}{ Muestra e=0,1 } \\
\hline Pampacocha & 509 & 85 & 254 & 103 & 52 & $\mathrm{M}$ & 38 & 19 & $\mathrm{M}$ \\
& & & & 51 & $\mathrm{~V}$ & & 19 & $\mathrm{~V}$ \\
Queral & 241 & 50 & 125 & 52 & 28 & $\mathrm{M}$ & 19 & 10 & $\mathrm{M}$ \\
& & & & & 24 & $\mathrm{~V}$ & & 9 & $\mathrm{~V}$ \\
Miramar & 208 & 43 & 106 & 44 & 23 & $\mathrm{M}$ & 16 & 8 & $\mathrm{M}$ \\
& & & & & 21 & $\mathrm{~V}$ & & 8 & $\mathrm{~V}$ \\
Chinchay & 117 & 16 & 61 & 25 & 13 & $\mathrm{M}$ & 9 & 5 & $\mathrm{M}$ \\
& & & & & 12 & $\mathrm{~V}$ & & 4 & $\mathrm{~V}$ \\
\hline Totales & 1075 & 194 & 546 & 224 & 116 & $\mathrm{M}$ & 82 & 42 & $\mathrm{M}$ \\
& & & & & 108 & $\mathrm{~V}$ & & 40 & $\mathrm{~V}$ \\
\hline
\end{tabular}

La investigación cuenta con la colaboración del grupo de comuneros varones, mayores de edad según el criterio de las comunidades, de un quinto caserío de «alpaqueros» (pastores de alpacas) denominado Cajabamba Alta, de la comunidad Cajabamba. El caserío Cajabamba Alta posee características distintas a las de los caseríos objetos de la intervención. Se sitúa en la vertiente oeste de la cordillera negra y se caracteriza por un alto grado de aislamiento. No se trata de una muestra representativa de la población del caserío toda vez que sólo se pudo trabajar con el grupo de varones 
descritos. Su inclusión supone la inclusión de un grupo de comuneros con reconocimiento público de funcionamiento excelente y la posibilidad de compararlo con los caseríos de la Comunidad Cruz de Mayo.

La Comunidad Cruz de Mayo carece de infraestructura básica: existe un grifo de agua por familia, no hay corriente eléctrica, las fuentes energéticas son las tradiciones, fundamentalmente el fuego. La infraestructura viaria se reduce a un camino afirmado, que llega hasta Pampacocha $(\mathrm{H}>>3.300 \mathrm{msnm})$ pasando por Miramar $(\mathrm{H}>>3.150 \mathrm{msnm})$ y Queral (H>> $3.200 \mathrm{msnm})$. A Chinchay (H>> $3.300 \mathrm{msnm})$ no llega la pista, se accede solo por camino de herradura desde Pampacocha durante 10 minutos. No hay línea regular de viajeros, la movilidad es privada. El transporte colectivo se activa a primera hora de la mañana cuando hay personas suficientes para rentabilizar la subida de un taxi de 5 plazas en el que suben 8 personas (en ocasiones hasta 12) o una "combi". Siempre se puede alquilar un taxi a nivel privado pero esta opción escapa económicamente de los miembros de la comunidad. En el invierno la duración del trayecto es de algo más de una hora, en tiempo de lluvias se incrementa considerablemente.

También se dispone de un camino (no apto para ningún tipo de vehículo) que supera los $1.200 \mathrm{~m}$ de desnivel entre Caraz y Pampacocha en apenas $8 \mathrm{Km}$. Este desnivel, junto a los cortados y su estrechez lo hacen un camino muy difícil para quien tenga falta de costumbre y no lo conozca. Un miembro de la comunidad joven y sano recorre el trayecto bajando en aproximadamente 45 minutos a una hora, tardando más de dos horas y media los maestros o un excursionista. La subida, confiesan los comuneros, se recorre en dos horas, los maestros apuntan que no suben andando pero en este caso les llevaría cerca de las cinco horas.

Existe un puesto de salud para los cuatro caseríos ubicado en Pampacocha y una escuela de primaria por caserío. La educación secundaria se realiza en Caraz y los alumnos acceden a los centros a pie y en las condiciones antes descritas.

Se ha utilizado el Cuestionario de Representación Organizacional $\left(\mathrm{CREO}^{\circ}\right)$ diseñado y validado por De Miguel (1999) traducido y adaptado al Quechua del callejón de Huaylas. La traducción y adaptación del $\mathrm{CREO}^{\circ}$ a la lengua Quechua del Callejón de Huaylas se ha desarrollado en tres fases: la primera de ellas ha sido idiomática, la segunda metodológica y la tercera connotativa.

\section{Idiomática:}

El profesor Víctor Laguna, lingüista de la Universidad Teológica ICI de USA, realizó la traducción. Se ha realizado la traducción y adaptación del cuestionario, en los siguientes momentos

a. traducción oral al quechua de los ítems y de las diferencias entre el $\mathrm{CREO}^{\mathrm{c}}$ y $\mathrm{CREO}^{\mathrm{s}}$,

b. identificación de definiciones aproximadas, relativas a conceptos de planificación, análisis y procesos sistemáticos de trabajo,

c. redacción de la traducción de los ítems, tomando en cuenta las definiciones aproximadas,

d. traducción oral del CREO quechua al español,

e.rectificación de la redacción al quechua, haciendo una aproximación conceptual y connotativa de los términos,

f. explicación y aplicación del cuestionario a dos comuneros bilingües. 
g. registro de los términos no comprendidos y de la conceptualización de éstos, h. redacción definitiva.

La traducción del cuestionario ha servido para generar nuevas hipótesis de trabajo y nuevos retos planteados por la significativa falta de conceptos en quechua relativos a la planificación, análisis y procesos sistemáticos de trabajo.

\section{Metodológica:}

La versión definitiva del CREO quechua resultó ineficaz para recoger la información. De hecho los 25 primeros cuestionarios de esta versión se rechazaron. Una gran parte de ellos se estaban contestando de forma mecánica y/o respondían a una lógica, hasta el punto que en ocasiones el encuestador podía adivinar la respuesta al ítem antes de realizar la pregunta.

Se tuvo, entonces, que realizar una adaptación metodológica en la que colaboró el profesor Dr. Luis Vicuña. Esta adaptación supuso:

- Modificación de la escala Likert de 5 a 4 alternativas de respuesta para eliminar la tendencia a la elección del punto medio, ya fuera por falta de comprensión o deseabilidad social.

- Variación de la fórmula original para recoger la información (cuestionario auto aplicada a recogida de datos por aplicadores).

- Aplicación simultanea de ambas escalas, para lograr la comprensión de las diferencias entre las escalas imagen-satisfacción y evitar el agotamiento por un tiempo prolongado de evaluación.

- Inclusión de 2 ejemplos (1 aplicado a la escala de creo cognición, y otro a la escala creo satisfacción).

- Utilización de recursos iconográficos como se muestra en 105 ejemplos siguientes: 


\section{CREO QUE EN MI PUEBLO LA GENTE ES HONRADA}

creemi que comunidanecho genticuna honraducayaptin

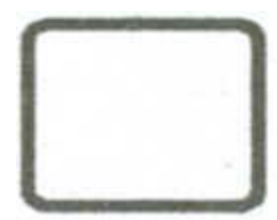

NADA

MANAM

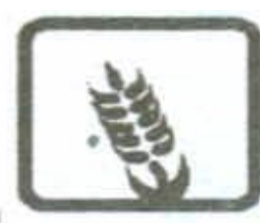

POCO

WALLCA

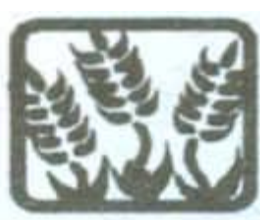

BASTANTE ATSKA

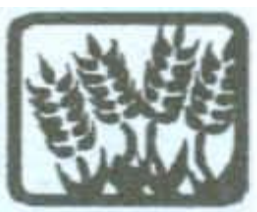

MUCHO

ALLAPA ATSKA

ESTOY A GUSTO CON LA HONRADEZ DE MI PUEBLO

\section{ALLAPA KUSHISHQAM QUE PUEBLACHO HONRADUCAYAPTIN}

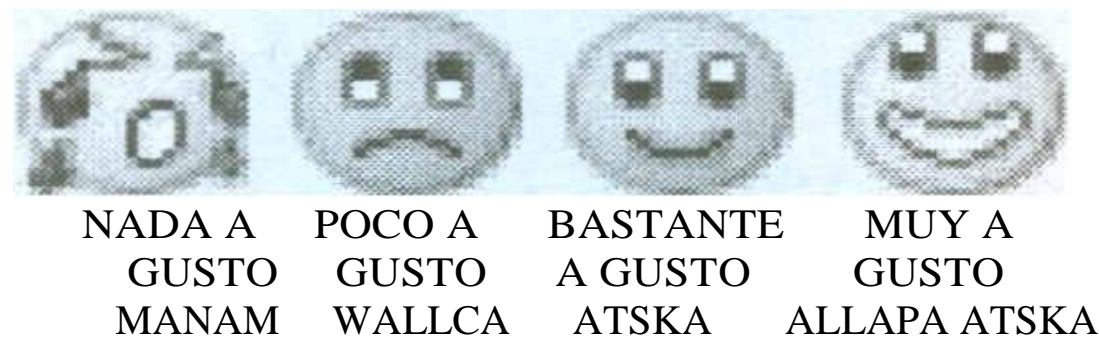

\section{Connotativa:}

Se han traducido los elementos del cuestionario al quechua, pero estos elementos correspondían a variables identificadas en un contexto profesional y laboral regido por la lógica del mercado capitalista. Por el contrario, las comunidades andinas se rigen por los principios ancestrales de reciprocidad y redistribución; su lógica de intercambio queda legislada en la Ley $n^{\circ} 24656$ de las comunidades campesinas.

La adaptación connotativa se realizó tras recoger la información y respondía a la necesidad de contrastar sobre qué concepto se estaba realmente interrogando. El objetivo de esta adaptación consistió en delimitar y definir las variables Creo Quechua. Se hizo mediante la traducción, de cada uno de los elementos que componen el cuestionario, del quechua al español y la adjudicación de un término en quechua a cada par de elementos que forman una variable. A continuación, se tradujo el término quechua de la variable al español. La adaptación connotativa se realizó mediante un acuerdo interjueces, actuando como jueces 3 miembros bilingües de la comunidad. Por último, se solicitó al traductor oficial que realizara la tarea de trasladar los términos quechuas de las variables al español, encontramos, en ocasiones, diferencias connotativas relevantes.

El proceso de adaptación (en sus tres momentos) no afectó a la función y estructura del instrumento. 


\begin{tabular}{|c|c|}
\hline VARIABIE & ITEMS \\
\hline 1. RETRIBLOOON & 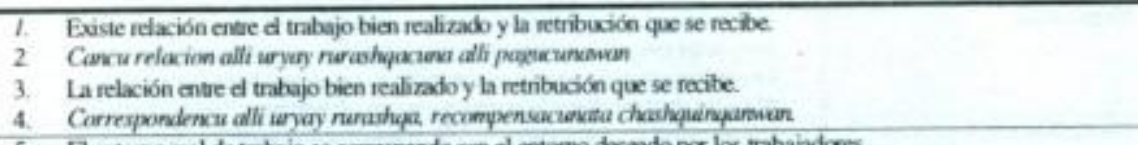 \\
\hline $\begin{array}{l}\text { 2. SATISFACOOONENE. } \\
\text { TRMBAO }\end{array}$ & 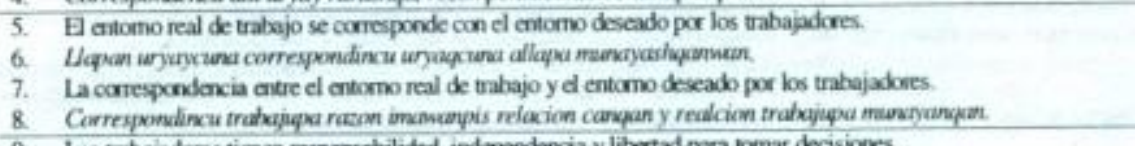 \\
\hline 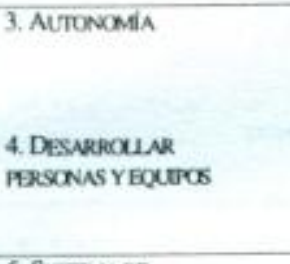 & 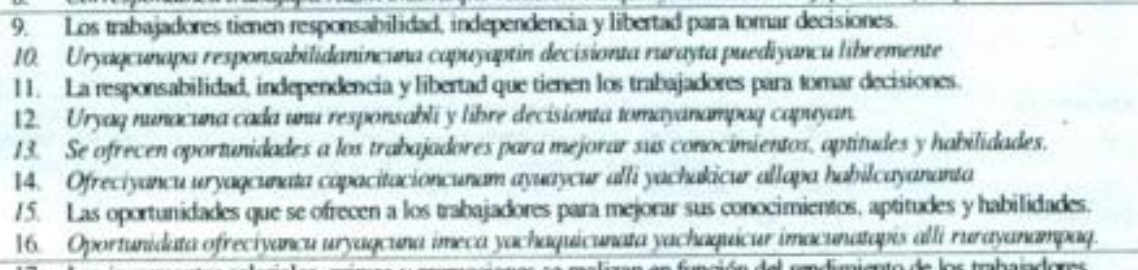 \\
\hline $\begin{array}{l}\text { 5. SISTEMADE } \\
\text { REOOMENSA }\end{array}$ & 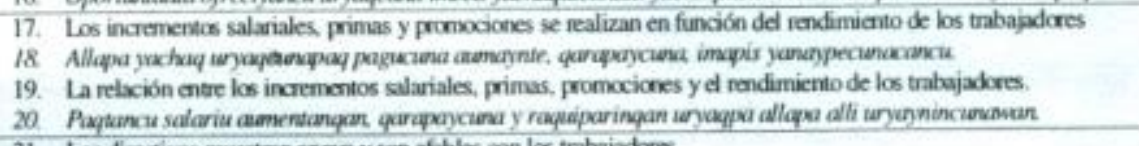 \\
\hline 6. APOYO & 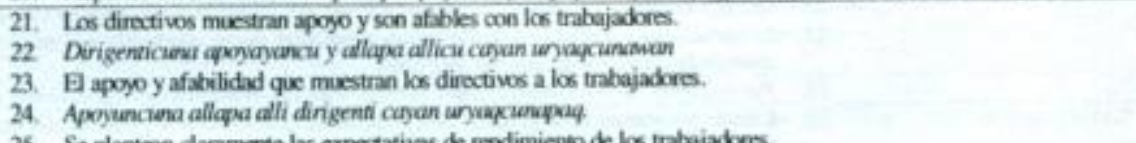 \\
\hline 7. Dikecaón & 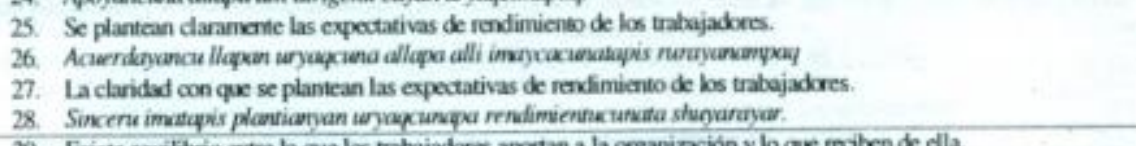 \\
\hline 8. EQUIDND & 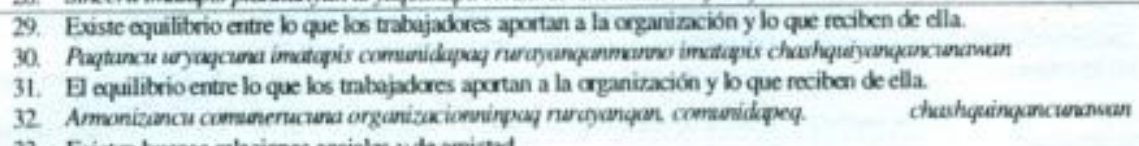 \\
\hline 9. RELACAONES SOCIALES & 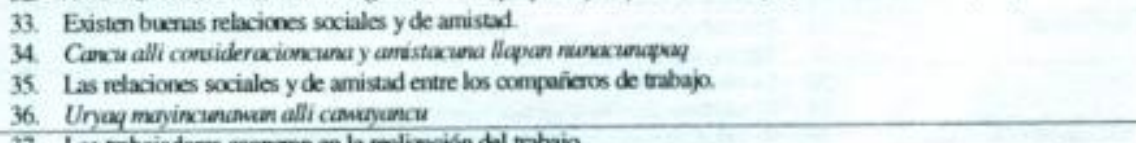 \\
\hline 10. COOOERACXÓN & 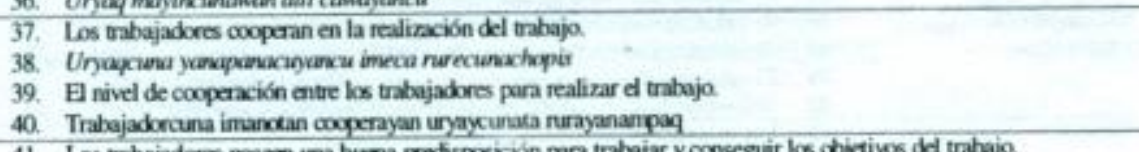 \\
\hline 11. MOTTVACÓ́N & 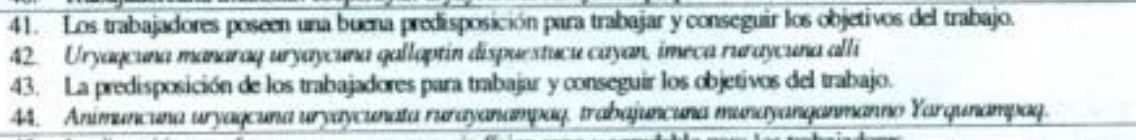 \\
\hline 12. CONPOKT & 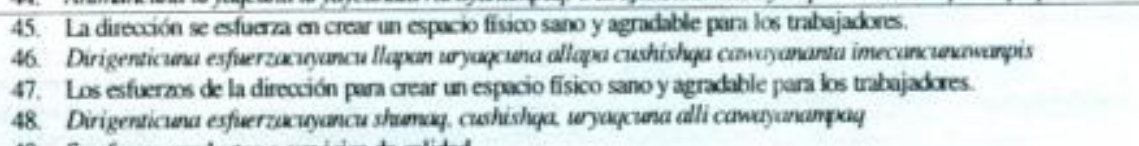 \\
\hline 13. CNIDND & 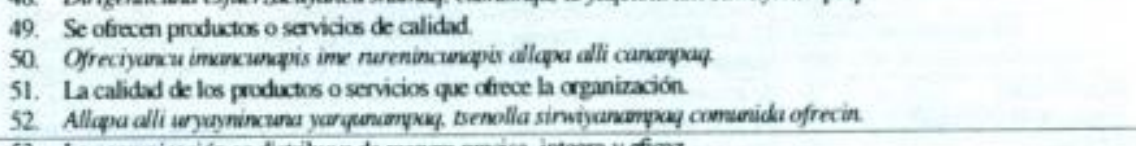 \\
\hline 14. COMENICACTÓN & 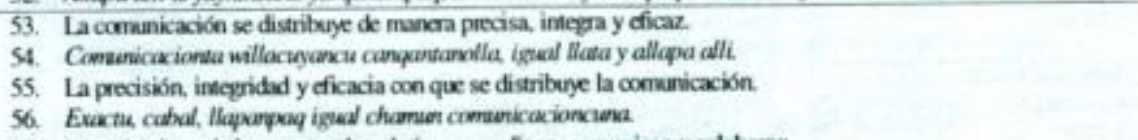 \\
\hline 15. COHESÓN & 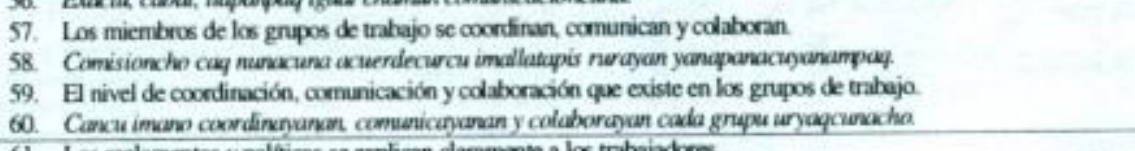 \\
\hline 16. QARUDAD & 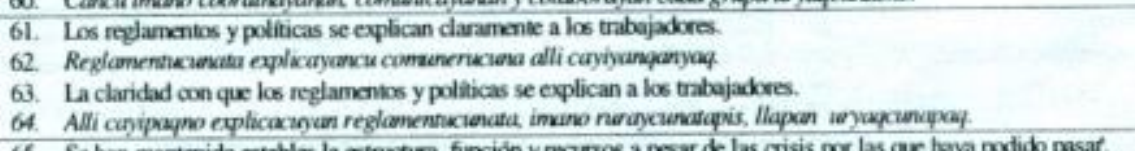 \\
\hline 17. ESTABHIDAD & 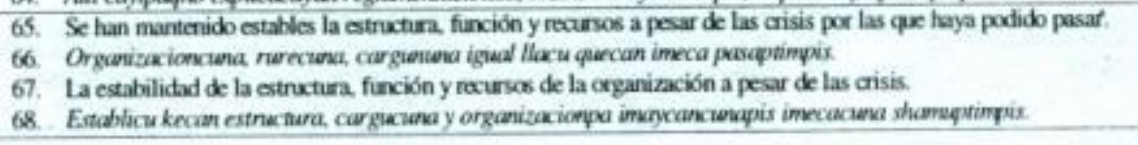 \\
\hline
\end{tabular}




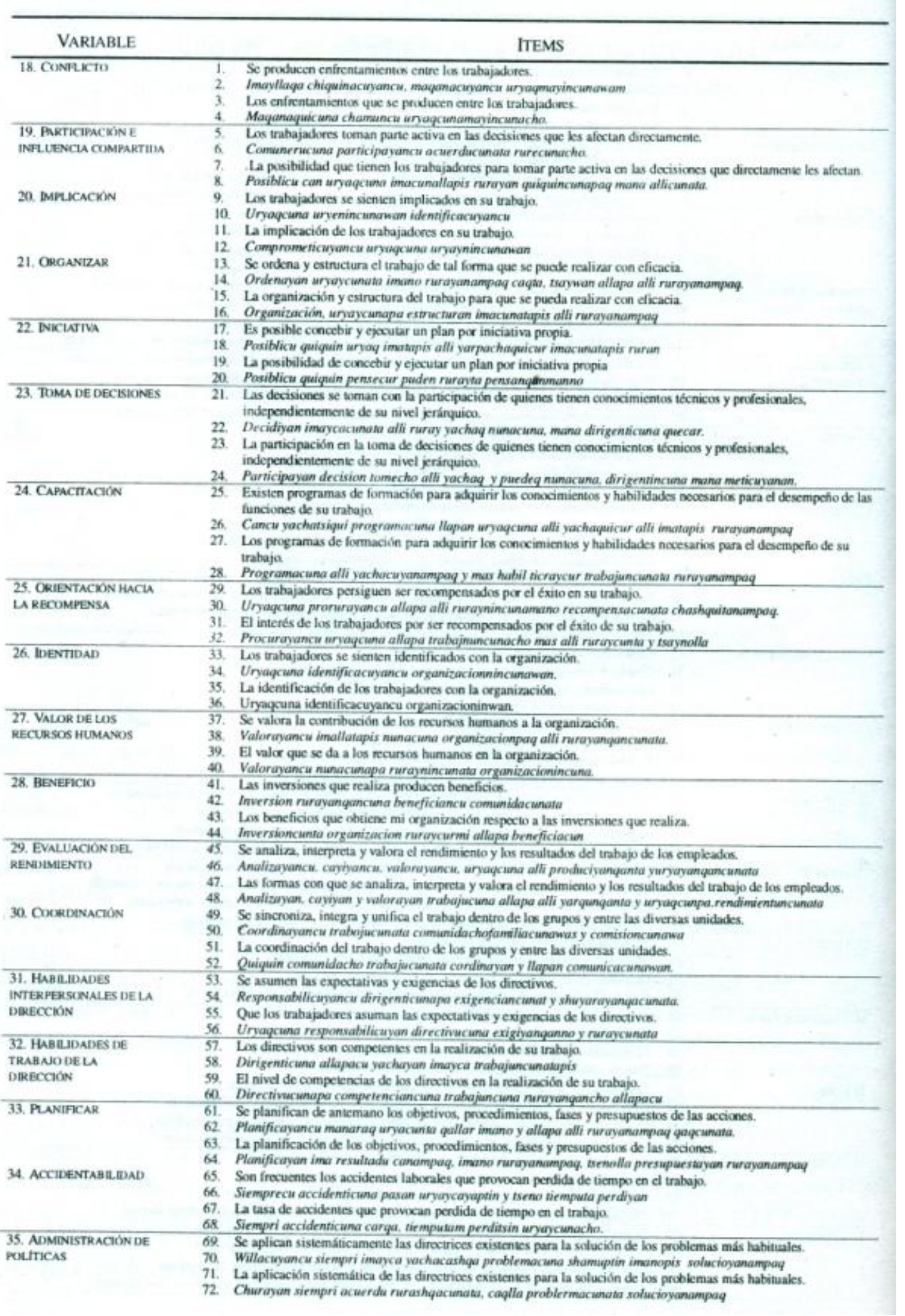




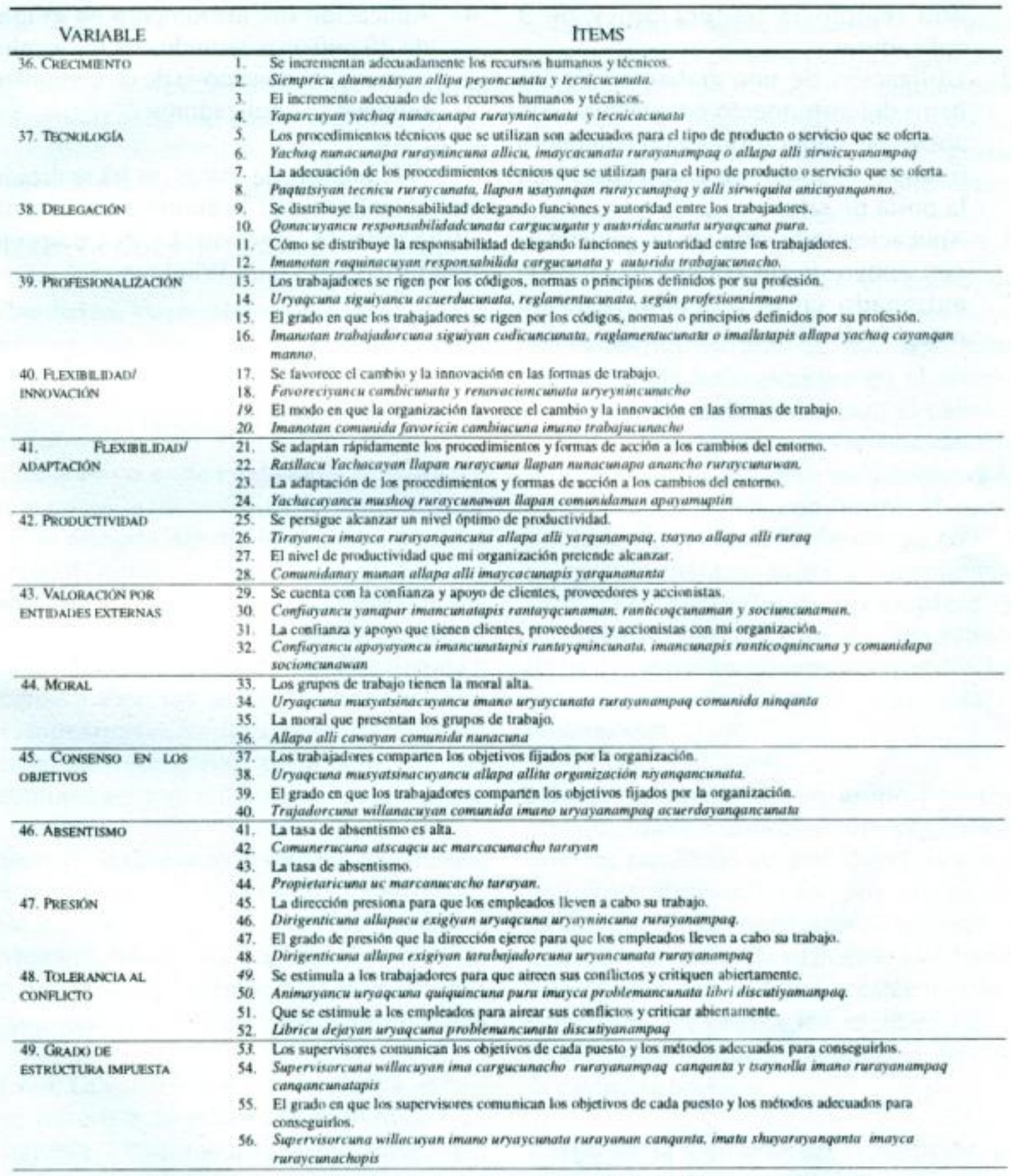

Tabla 3. Relación de variables e items incluidos en el CREO

La recogida de datos comenzó en febrero de 2003 y se concluyó en septiembre del mismo año. La estrategia para recoger los protocolos se diversificó en las siguientes fórmulas:

1. Reunión con los dirigentes y comuneros para la aplicación piloto de cuestionario, con ayuda del lingüista que realizó la traducción, y de 3 aplicadores.

2. Utilización de una grabación de los ítems del instrumento en quechua, con apoyo de 3 aplicadores y un comunero bilingüe, reunidos en grupos de 10 en la posta de salud. 
3. Aplicación individual en las viviendas; con apoyo de un comunero bilingüe entrenado en el procedimiento de recogida de información y un aplicador.

4. Aplicación del instrumento en grupos de 20 personas reunidos en los locales comunales, con apoyo de un comunero bilingüe y 2 aplicadores.

El análisis de datos se ha realizado en la Universidad Autónoma de Madrid utilizando la versión 11 del paquete estadístico SPSS para Windows. 


\section{RESULTADOS}

La interpretación de los resultados obtenidos se ha realizado considerando las variables contextuales que suponen la redacción de las hipótesis 2 a a 2i.

\section{Las Variables de Estudio}

Condición; Hace referencia a la relación y estatus que mantiene la persona con la comunidad y la posesión de la tierra. Según J. C. Mariátegui desde tiempo del Ayllu el reconocimiento de la condición de Comunero o el de ciudadanía se otorga al que posee la tierra. Posteriormente a partir de la reforma Agraria, esta condición dio un giro, puesto que se realizó un proceso de expropiación y repartición de los latifundios (haciendas) en donde los indios o sirvientes pasaban a poseer $<<$ las tierras $>>y$ conformaban las llamadas «comunidades campesinas». Se han considerado las siguientes categorías: comunero, hijo de comunero y propietario

Sexo; Se incluye como variable dicotómica teniendo en cuenta la estructura biológica de la persona para delimitar las categorías Mujer/Hombre. Esta variable determina la posibilidad de accesos a la educación y participación en la representación comunal

Edad; La variable sociodemográfica «edad» se refiere a la edad del individuo que contesta al instrumento y no a la antigüedad de la comunidad. Su inclusión obedece a, criterios de índole física, estableciéndose como categorías: de 16 a 35 años y mayores de 35 años con base en criterios metodológicos de muestreo (puesto que rangos diferentes, concentrarían estratos de edad de tamaño reducido que impedirían comparaciones estadísticas.

Estado Civil: Referido a la situación jurídica concreta que la persona ocupa o guarda en relación con la familia, y la comunidad; pero que no establece diferencias en las obligaciones y capacidades. Las categorías incluidos han sido: casado/a, conviviente, separado/a, soltero/a y viudo/a.

Nivel de Educación: Es el grado de instrucción adquirida en instituciones de educación formal básica, que determina facultades de lecto-escritura en el idioma español, y las habilidades para el cálculo.

Para las categorías incluidas en esta variable es importante si el sujeto tuvo acceso a la educación o si ha concluido el nivel primaria y/o secundario. Se cuenta con las categorías: iletrado, primaria incompleta, primaria, secundaria incompleta y secundaria, técnica y superior. Los sujetos de la muestra se encontraban entre las categorías: iletrado y secundaria incompleta.

Idioma: Lenguaje de un grupo humano (comunidad) categorizado según las diversas facultades para .el Quechua y/o el español, determinado por nivel de educación al que tiene acceso el sujeto y que a su vez posibilita su interacción dentro y fuera de la comunidad. Las categorías han sido: habla, lee y habla, lee, escribe y habla. Para el idioma español además se incluyó la categoría no sabe.

Cargo en la Comunidad: Condición de confianza y responsabilidad, asignada y reconocida por los miembros de la comunidad que recae en un individuo, con la finalidad de realizar tareas determinadas (asignación de tareas comunales, juez de paz, etc.) y 
representar al grupo. Reconocidos con el nombre de comisionados en la comunidad Cruz de Mayo. Las alternativas incluidas son: con cargo y sin cargo.

\section{Análisis Descriptivo}

Respecto al CREOc, un escaso número de variables superan la puntuación de 3 (bastante) y lo hacen de forma poco llamativa: productividad es la variable más valorada, llega a obtener una puntuación media de 3,15. La tendencia a la respuesta en los valores medios de la escala mostrada por los sujetos se sigue manifestando a pesar de reducir la escala original a una escala de 4 puntos. Identidad $(\mathrm{x}=3,08)$, Coordinación $(=3,05)$, y presión $(=3,01)$. Valor de los recursos humanos, relaciones sociales, motivación, participación, grado de estructura impuesta, comunicación y cooperación obtienen puntuaciones medias superiores a 2,8. A falta de un análisis de agrupación de variables encontramos evidencias teóricas que nos permiten afirmar que existe un factor común entre ellas: se trata de variables de gestión, aquellas que en las organizaciones empresariales dominan y controlan los gerentes para propiciar equipos eficaces de producción: Productividad (3,16), Identidad (3,08), Coordinación 3,05), Presión 3,01, Valor de los RR.HH. (2,99), Relaciones sociales (2,96), Motivación (2,94), Participación $(2,87)$, Grado de estructura impuesta $(2,86)$, Comunicación $(2,85)$, Cooperación $(2,81)$, Evaluación del rendimiento $(2,80)$.

De esta manera 9 de las 49 variables no alcanzan una puntuación media de 2,5: Retribución (2,14), Conflicto (2,20), Toma de decisiones (2,29), Equidad (2,32), Accidentabilidad (2,32), Satisfacción general $(2,36)$, Desarrollo personal $(2,46)$, Apoyo de la dirección $(2,47)$, Valoración por Ent. Ext. $(2,49)$

Respecto al CREOs los comuneros se muestran más generosos cuando se trata de valorar su nivel de satisfacción con las variables de la escala. En este caso son 9 las variables cuya puntuación media supera el grado de bastante (3), Identidad $S(3,17)$, Productividad S $(3,14)$, Relaciones sociales S $(3,12)$, Motivación S $(3,12)$, Iniciativa S $(3,06)$, Coordinación S $(3,05)$, Valor de los RR.HH. S $(3,04)$, Comunicación S $(3,02)$, Grado de estructura impuesta S (3,01), Organizar S (2,98), Cohesión S $(2,93)$, Implicación S (2,92), Evaluación del rendimiento $S(2,91)$, Moral $S(2,90)$, Confort $S(2,88)$, Cooperación S (2,87), Autonomía toma decisiones S (2,86), Flexibilidad/Innovación S (2,85), Habilidades inter-pers. Direc. S $(2,83)$, Flexibilidad/Adaptación S $(2,81)$, Presión S $(2,81)$, Planificar S $(2,81)$, Sistema de recompensas S $(2,81)$, Claridad S $(2,81)$,

A pesar de que la media de las medias de las puntuaciones alcanzadas en la escala de satisfacción supera a la de cognición, se aprecia mayor poder de discriminación, con puntuaciones más bajas, aunque solo cuatro variables se sitúan en una media igual o inferior a 2,5.La más significativa es la variable participación: Participación $S(2,50)$, Apoyo de la dirección S $(2,47)$, Conflicto S $(1,99)$, Accidentabilidad S $(1,85)$

\section{Contrastación de Hipótesis}

\section{HIPÓTESIS 1}

La imagen (representación compartida y socialmente construida) que tienen los comuneros de su comunidad está determinada por el objeto representado (comunidad) es el 
supuesto que plantea la hipótesis 1:

Hipótesis 1: La imagen de la comunidad es propia de cada comunidad Hipótesis 1 a: La imagen de dos comunidades distintas es distinta.

Hipótesis 1 b: Mediante la imagen se puede predecir la pertenencia de un individuo a una determinada comunidad

\section{comunidades:}

\begin{tabular}{|c|c|c|c|c|c|}
\hline \multicolumn{6}{|l|}{ Autovalores } \\
\hline \multirow[t]{2}{*}{ Función } & & Autovalor & $\%$ de varianza & $\%$ acumulado & Correlación canónica \\
\hline & 1 & 3,16828577 & 100 & 100 & 0.87183325 \\
\hline $\mathbf{a}$ & & \multicolumn{4}{|c|}{ Se han empleado las 1 primeras funciones discriminantes canónicas en el análisis. } \\
\hline \multicolumn{6}{|l|}{ Lambda de Wilks } \\
\hline \multirow[t]{2}{*}{ Contraste de las funciones } & & $\begin{array}{l}\text { Lambda de } \\
\text { Wilks }\end{array}$ & Chi-cuadrado & gl & Sig. \\
\hline & 1 & 0.239906776 & 105,63536 & 10 & $4.03630 \mathrm{E}-18$ \\
\hline
\end{tabular}

Coeficientes estandarizados de las funciones discriminantes canónicas

Función

Satisfacción general

$0, \overline{4} 93558873$

Dirección

0.369328228

Equidad

0,522951557

Relaciones sociales

$-0.560570914$

Cooperación

0,714986879

Calidad

$-0,528404949$

Implicación

0,369564613

Beneficio

0,458483906

Accidentabilidad

$-0,28900381$

Absentismo

$-0,967831578$

Funciones en los centroides de los grupos

Comunidad

Función

Cruz de Mayo

Cajabamba

4,68395144

Funciones discriminantes canónicas no tipificadas evaluadas en las medias de los grupos

Resultados de la clasificación

\begin{tabular}{|c|c|c|c|c|c|}
\hline \multirow{6}{*}{ Original } & & \multirow{3}{*}{$\begin{array}{l}\text { Comunidad } \\
\text { Cruz de Mayo }\end{array}$} & \multicolumn{2}{|c|}{ Grupo de pertenencia pronosticado } & \multirow[t]{2}{*}{ Total } \\
\hline & \multirow{3}{*}{ Recuento } & & Cruz de Mayo & Cajabamba & \\
\hline & & & 80 & 1 & 81 \\
\hline & & Cajabamba & 1 & 10 & 11 \\
\hline & $\%$ & Cruz de Mayo & 98,7654321 & 1.234567901 & 100 \\
\hline & & Cajabamba & 9,090909091 & 90,90909091 & 100 \\
\hline
\end{tabular}

Clasificados correctamente el $97.8 \%$ de los casos agrupados originales.

Tabla 2. Resultados del análisis discriminante 
Los coeficientes estandarizados de las suscribiendo que la imagen de dos funciones discriminantes canónicas indican comunidades distinta es distinta, toda vez la peculiaridad de cada comunidad, que podemos aislar una serie de variables coeficientes estandarizados de las funciones discriminantes canónicas indican la peculiaridad de cada comunidad, suscribiendo que la imagen de dos comunidades distinta es distinta, toda vez que podemos aislar una serie de variables que son representativas de una comunidad respecto a su comparación con otra.

De esta manera se pueden afirmar que resulta representativa para Cajabamba Alta respecto a Cruz de Mayo una mayor valoración de: satisfacción general, dirección, equidad, cooperación, implicación y beneficio. Resulta discriminativa para Cruz de Mayo respecto a Cajabamba Alta una mayor valoración de: relaciones sociales, calidad, accidentabilidad y absentismo.

Los resultados de clasificación de los sujetos son alentadores: En este análisis se considera, por un lado, la comunidad a la que pertenecen los sujetos que han participado en el estudio (81 de Cruz de Mayo y 11 de Cajabamba). Por otro lado, un pronóstico sobre la comunidad a la que deberían pertenecer los sujetos considerando las respuestas que-han dado en el cuestionario CREOc, esto es, la imagen que han reflejado de su comunidad.

El análisis tiene capacidad para pronosticar acertadamente la pertenencia a una u otra comunidad de 90 de los 92 casos considerados, esto es, al 97,8\% de los sujetos se le ha asignado correctamente la comunidad a la cual pertenecen considerando la imagen manifestada mediante la aplicación del cuestionario. Imaginemos que encontramos en Caraz un comunero de una de las dos comunidades y le pedimos que conteste al CREOc. Mediante la imagen que nos ha trasmitido de su comunidad al responder al CREOc, se podría pronosticar (con un $97,8 \%$ de probabilidad de acierto) a qué comunidad pertenece.

Este resultado resulta especialmente interesante: con esta capacidad de clasificación y predicción podemos afirmar que el cuestionario CREO quechua resulta un instrumento válido para analizar la representación colectiva que comparten los comuneros sobre su comunidad.

\subsection{HIPÓTESIS 2.}

La imagen se genera por y en la interacción que se produce entre los sujetos de la organización y la interacción está posibilitada por la posición que tienen las personas en la comunidad. Posición definida por cualquier condición social, algunas de ellas son las que hemos incluido en el estudio como variables contextuales, y las categorías incluidas en tales variables delimitan las posiciones analizadas. Este es el supuesto que esboza la redacción de la hipótesis 2:

Hipótesis 2: La imagen se modula por la posición que ocupan los comuneros en la comunidad. Por ello encontraremos diferencias en la imagen en función de la posición ostentada.

Hipótesis 2a: La imagen está modulada por la pertenencia a un caserío

Hipótesis 2b: La imagen está modulada por la condición

Hipótesis 2c: La imagen está modulada por el sexo

Hipótesis 2d: La imagen está modulada por la edad

Hipótesis 2e: La imagen está modulada por el estado civil

Hipótesis 2f: La imagen está modulada por el nivel de estudios. 
Hipótesis 2g: La imagen está modulada por el manejo del idioma quechua Hipótesis $2 \mathrm{~h}$ : La imagen está modulada por el manejo del idioma español Hipótesis 2i: La imagen está modulada por tener un cargo en la comunidad

La hipótesis 2 se ha contrastado en la comunidad Cruz de Mayo. La posición que ocupan los sujetos en la comunidad ha sido definida por las variables contextuales definida en el punto 5.1., definiendo cada una de estas variables una de las subhipótesis planteadas. La tabla siguiente presenta las diferencias encontradas.

\begin{tabular}{|c|c|c|c|c|c|c|c|c|c|}
\hline VARIABLE & $\begin{array}{l}\frac{0}{\alpha} \\
u \\
u \\
\vdots \\
u\end{array}$ & $\begin{array}{l}\frac{2}{4} \\
\text { o } \\
\text { z } \\
0 \\
u\end{array}$ & 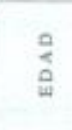 & $\begin{array}{l}\stackrel{0}{x} \\
\text { w } \\
\text { w }\end{array}$ & 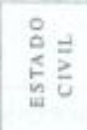 & 号 & 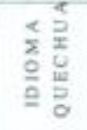 & 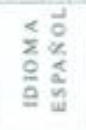 & $\begin{array}{l}0 \\
\vdots \\
\vdots \\
\vdots\end{array}$ \\
\hline \multicolumn{10}{|l|}{ 1. RETRIBUCION } \\
\hline 2. SATISFACCION EN EI. TEABAID & & & & & 0.029 & & & & \\
\hline 3. AVTONOMIA & & 0.033 & & & & & & & \\
\hline $\begin{array}{l}\text { 4. DESARKDLLAR FERSONAS Y } \\
\text { EQUTFOS }\end{array}$ & 0,000 & & & & & & & & \\
\hline 5. SISTEMA DERECOMPENSA & 0,005 & & & & & & & & \\
\hline 6. Aroyo & 0,023 & & & & & & & & \\
\hline 7. DIRECCHON & & & & & & & & & 0.025 \\
\hline 8. EQUUBBAD & 0,000 & & & & & & & & \\
\hline 9. RELLACIONES SOCIALES & & & 0,019 & & & 0.019 & 0,016 & & \\
\hline 10. COOPERACION & 0,034 & & & & & & & & \\
\hline 11. Motivacion & 0.019 & & & & & 0.037 & 0,007 & & \\
\hline \multicolumn{10}{|l|}{ 12. CONFONT } \\
\hline 13. CALIDAD & 0,005 & & & & & & & & \\
\hline 14. COMUNICACTON & & & & 0.010 & & 0.002 & & & \\
\hline 15. Conrsion & 0.014 & 0.037 & & & & & & & \\
\hline 16. CLAN16A0 & 0.004 & & & & & & & 0,012 & \\
\hline \multicolumn{10}{|l|}{ 17. ESTABII.18AD } \\
\hline 18 CONHLICTO & & $c$ & & & & & & & \\
\hline $\begin{array}{l}\text { 19. PARTICIFACION E INTLUENCIA } \\
\text { COMPARTBOA }\end{array}$ & 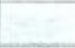 & & & & 0.019 & & & & 0.042 \\
\hline 20. IMNTICACION & 0.033 & 0.002 & & & & & & & \\
\hline 21. ORCANIYAR & & & & & & 0,017 & & & \\
\hline \multicolumn{10}{|l|}{ 22. INICLATIVA } \\
\hline \multicolumn{10}{|l|}{ 23. TOMA DE DECISIONES } \\
\hline 24. CAPACITACHOS & 0,000 & & & & & & & & \\
\hline $\begin{array}{l}\text { 29. ORIENTACIOS WACIALA } \\
\text { KECOMPENSA }\end{array}$ & & & & 0.050 & & & & & \\
\hline 26 IOENTIDAD & & & & 0.003 & & & & & 0.046 \\
\hline \multicolumn{10}{|l|}{$\begin{array}{l}\text { 27. VALOR DE LOS KECURSOS } \\
\text { HUMANOS }\end{array}$} \\
\hline 28. BENEFICLO & 0,000 & & & & & 0.003 & & & \\
\hline 29. EVALUACION DEL RENDIMIENTO & & 0.019 & & & 0.036 & & & & \\
\hline 30. COOKDINACION & 0.003 & & & & & & & & \\
\hline \multicolumn{10}{|l|}{$\begin{array}{l}\text { 31. HADHLIDADES INTHR PERSOSALES } \\
\text { DE LA DIRECCLON }\end{array}$} \\
\hline $\begin{array}{l}\text { 32. HABILIDABES DE TRABAFO BELA } \\
\text { DIKFCCBOA }\end{array}$ & & & & & 0.009 & & & & \\
\hline 33. PLAXIFICAR & & & & 0.038 & & & & & \\
\hline 34. ACCIDENTABIIIDAD & & & & & 0.037 & & & & \\
\hline \multicolumn{10}{|l|}{ 35. ADM INISTRACION DE POLLITICAS } \\
\hline 36. CEFCIMIENTO & & & & & & & $=$ & & \\
\hline 37. TECNOIDOKA & 0,000 & & & & & & & & \\
\hline 38. DULLEACION & & & & & & & & & \\
\hline 39. PROFESHONALIZACIOK & 0.016 & & & & & & & & \\
\hline \multicolumn{10}{|l|}{ 40. FLEXIBILIDAD/ INNOVACION } \\
\hline A1 FLIXXIDILIDAD/ADAVTACION & 0,026 & & & 0,013 & & & & & \\
\hline \multicolumn{10}{|l|}{ 42. ProbECTIVIBAD } \\
\hline \multicolumn{10}{|l|}{$\begin{array}{l}\text { 43. VALOKACION FOK ENTIOADES } \\
\text { IKTIRNAS }\end{array}$} \\
\hline 44. MORAL. & 0.011 & & & & & & & & \\
\hline 45. CONSENSO EN LOS OALATIVOS & & & & 0,002 & 0.025 & & & & \\
\hline 46. ABSENTISMO & 0,000 & & & 0.037 & & & & & \\
\hline 47. PELSTON & & & & & 0.012 & & & & \\
\hline 48. TOLERANCLA AL. CONFLKTO & 0.002 & & & & & & 0.026 & & \\
\hline $\begin{array}{l}\text { 49. GRADO DE ESTRECTEEAA } \\
\text { MPUESTA }\end{array}$ & 0.007 & & & & & & & 9,000 & \\
\hline
\end{tabular}

Tabla 3. Diferencias estadísticamente significativas. Chi cuadrado de Pearson. Significación al 0.95 . 
La pertenencia a un caserío es la variable con mayor poder de discriminación, encontrando diferencias estadísticamente significativas en 20 de las 49 variables que estructuran el CREO. Encontramos evidencias de que la representación organizacional de la comunidad Cruz de Mayo está modulada por la pertenencia a uno u otro caserío.

Variables

4. DESARROLLAR PERSONAS Y EQUIPOS

8. EQUIDAD

24. CAPACITACIÓN

28. BENEFICIO

37. TECNOLOGIA

46. ABSENTISMO

48. TOLERANCIA ALCONFLICTO|

30. COORDINACIÓN

16. CLARIDAD

5. SISTEMA DE RECOMPENSA

13. CALIDAD

49. GRADO DE ESTRUCTURA IMPUESTA

44. MORAL

15. COHESIÓN

39. PROFESIONALIZACIÓN

11. MOTIVACIÓN

6. APOYO

41. FLEXIBILIDAD/ADAPTACIÓN

20. IMPLICACIÓN

10. COOPERACIÓN

\section{Caserio}

0,000

0,000

0,000

0,000

0,000

0,000

0,002

0,003

0.004

0.005

0.005

0.007

0.011

0.014

0.016

0.019

0.023

0.026

0.033

0.034

Las diferencias encontradas en las otras variables de agrupación son mucho menores: en estado civil y sexo se hayan diferencias en 7 variables. Posteriores análisis nos podrán detallar las características propias de cada uno de los grupos.

VARIABLE

45. CONSENSO EN LOS OBJETIVOS

26. IDENTIDAD

14. COMUNICACIÓN

41. FLEXIBILIDAD/ ADAPTACIÓN

46. ABSENTISMO

33. PLANIFICAR

25. ORIENTACIÓN HACIA
SEXO

0,002

0,003

0,010

0,013

0,037

0,038

0,050 
VARIABLE

32. HABILIDADES DE TRABAJO

DE LA DIRECCIÓN

47. PRESIÓN

19. PARTICIPACIÓN E INFLUENCIA

COMPARTIDA

45. CONSENSO EN LOS OBJETIVOS

2. SATISFACCIÓN EN EL TRABAJO

29. EVALUACIÓN DEL RENDIMIENTO

34. ACCIDENTABILIDAD
Estado Civil

0,009

0,012

0,019

0,025

0,029

0,036

0,037

Nivel estudios, condición, cargo y el dominio de los idiomas quechua y español resulta poco significativo, encontrando las diferencias que a continuación se detallan

VARIABLE

14. COMUNICACIÓN

28. BENEFICIO

21. ORGANIZAR

9. RELACIONES SOCIALES

11. MOTIVACIÓN

VARIABLE

20. IMPLICACIÓN

29. EVALUACIÓN DEL RENDIMIENTO

3. AUTONOMIA

15. COHESIÓN

VARIABLE

7. DIRECCIÓN

19. PARTICIPACIÓN

E INFLUENCIA COMPARTIDA

26. IDENTIDAD

\section{ESTUDIOS}

0.002

0.003

0.017

0.019

0.037

\section{CONDICIÓN}

0.002

0.019

0.033

0.037

\section{CARGO}

0.025

0.042

0.046
VARIABLE

11. MOTIVACIÓN

9. RELACIONES SOCIALES

48. TOLERANCIA AL CONFLICTO
IDIOMA QUECHUA

0.007

0.016

0.026 
VARIABLE

49. GRADO DE ESTRUCTURA

IMPUESTA

16. CLARIDAD
IDIOMA ESPAÑOL

0.000

0.012

Doce variables han quedado al margen de las diferencias encontradas entre los grupos definidos por las variables contextuales

\section{VARIABLES}

1. RETRIBUCIÓN

12. CONFORT

17. ESTABILIDAD

22. INICIATIVA

27. VALOR DE LOS RECURSOS HUMANOS

31. HABILIDADES INTERPERSONALES DE LA DIRECCIÓN

35. ADMINISTRACIÓN DE POLÍTICAS

36. CRECIMIENTO

38. DELEGACIÓN

40. FLEXIBILIDAD/ INNOVACIÓN

42. PRODUCTIVIDAD

43. VALORACIÓN POR ENTIDADES EXTERNAS 


\section{CONCLUSIONES ${ }^{1}$}

La hipótesis 1 ha sido contrastada encontrando que cada comunidad construye y comparte una representación de la comunidad que es sui géneris.

Se ha encontrado una función discriminante que clasifica correctamente a los sujetos de una comunidad u otra y lo hace con una altísima probabilidad de acierto $(97,8 \%)$.

Considerando esta capacidad de discriminación, el cuestionario CREO, versión quechua, ha sido validado para su aplicación en las comunidades de la zona de influencia de Caraz.

La pertenencia a un caserío se manifiesta como la variable de agrupación con mayor poder para diferenciar la imagen que tienen los miembros de la comunidad. El hecho de que se encontraran mayor número de variables que mostraban diferencias estadísticamente significativas respecto al caserío (diferencias en 20 variables) que a la comunidad (diferencias en 11 variables) llevo a realizar análisis discriminantes con el resultado de la correcta clasificación del $100 \%$ de los sujetos.

\section{Resultados de la clasificación}

\begin{tabular}{|c|c|c|c|c|c|c|}
\hline & \multirow[b]{2}{*}{ Caserío } & \multicolumn{4}{|c|}{ Grupo de pertenencia pronosticado } & \multirow[b]{2}{*}{ Total } \\
\hline & & Pamoacocha & Queral & Miramar & Chinchav & \\
\hline \multirow[t]{4}{*}{ Original Recuento } & Pampacocha & 34 & 0 & 0 & 0 & 34 \\
\hline & Queral & 0 & 15 & 0 & 0 & 15 \\
\hline & Miramar & 0 & 0 & 14 & 0 & 14 \\
\hline & Chinchay & 0 & 0 & 0 & 8 & 8 \\
\hline \multirow[t]{4}{*}{$\%$} & Pampacocha & 100,0 & , 0 & 0 & 0 & 100,0 \\
\hline & Queral & , & 100,0 & ,0 & 0 & 100,0 \\
\hline & Miramar & 0 & ,0 & 100,0 & , 0 & 100,0 \\
\hline & Chinchay & 0 & 0 & , 0 & 100,0 & 100,0 \\
\hline
\end{tabular}

\section{a. Clasificados correctamente el $\mathbf{1 0 0 , 0 \%}$ de los casos agrupados originales.}

El caserío posibilita y por ello determina, en mayor medida que cualquier otro motivo, la interacción entre los miembros de la comunidad. Las condiciones de vida, especialmente las infraestructuras no propician una comunicación continua y fluida, solo la proximidad espacial y la asistencia a las reuniones locales que se realizan en los caseríos favorecen el intercambio intersubjetivo que cimienta la representación compartida y socialmente construida de la comunidad.

La imagen de la comunidad está fuertemente arraiga entre el resto de los grupos que se han evaluado. Si bien el diagnóstico nos permite pronosticar quien es quien a muy alto nivel (sexo es la variable que peor pronostica la pertenencia a un grupo -hombre y mujer- y lo hace con un $90 \%$ de probabilidad de acierto), el bajo número de variables donde se han hallado diferencias nos permite comentar que existe un alto índice de identidad con la comunidad, o según los indicios anteriores con el caserío.

${ }^{1}$ Se esta elaborando un informe de conclusiones que incluye una reflexión estratégica para la comunidad cuyo contenido no se presenta en el informe científico para salvaguardar la confidencialidad del proyecto. 


\section{REFERENCIAS}

1. De Miguel, J. M. (2004). Dimensiones psicosociales del desarrollo local. En: De la Corte, L; Blanco, A. y Sabucedo; Psicología y Derechos Humanos. Madrid: Icaria.

2. De Miguel, J.M. (1999). "La Organización como construcción social Representación organizacional» Tesis Doctoral, Universidad Autónoma de Madrid, Facultad de Psicología, Dpto. Psicología Social y Metodología, Madrid, España.

3. Paredes, M. y González del Río, C. (2000) "Importancia de los valores andinos para una Gestión de Calidad" Revista de la Facultad de Psicología de la-Universidad Nacional Mayor de San Marcos, Año IV, Nº, Lima, Perú, pp 91-110.

4. Paredes, M. (1994) "Gestión Empresarial: Factor de Competitividad en el Comercio Internacional» Universidad Nacional de San Cristóbal de Huamanga, Lluvia Editores.1era. Ed. Lima, Perú.

5. González del Río, C. y Paredes, M. (1999) "Cultura y percepción de la realidad» Revista de la Facultad de Psicología de la Universidad Nacional Mayor de San Marcos, Año III, N4, Lima, Perú, pp123-137. 